\title{
Contaminación bacteriológica en teléfonos celulares de trabajadores de la salud en ambiente clínico: revisión sistemática
}

\section{Bacteriological contamination in cell phones of health workers in a clinical setting: a systematic review}

\author{
Yeny Zulay Castellanos-Domínguez ${ }^{1}$ (D) , María Cecilia Cruz ${ }^{2}$ (D) , Ludín Thalía Jiménez ${ }^{3}$ iD) Jorge Armando Solano ${ }^{4}$ (iD \\ 1. Universidad Santo Tomás. Bucaramanga, Colombia. Correo: yeny.castellanos@ustabuca.edu.co - https://orcid.org/0000-0001-5881-1998 \\ 2. Universidad Santo Tomás. Bucaramanga, Colombia. Correo: maria.cruz01@ustabuca.edu.co - https://orcid.org/0000-0002-8601-1776 \\ 3. Universidad Santo Tomás. Bucaramanga, Colombia. Correo: ludin.jimenez@ustabuca.edu.co - https://orcid.org/0000-0002-4991-0027 \\ 4. Universidad Santo Tomás. Bucaramanga, Colombia. Correo: jorge.solano@ustabuca.edu.co - https://orcid.org/0000-0003-0967-1698 \\ Tipología: Artículo de revisión \\ Para citar este artículo: Castellanos-Domínguez YZ, Cruz MC, Jiménez LT, Solano JA. Contaminación bacteriológica en teléfonos celulares de trabajadores de la salud en ambiente \\ clínico: una revisión sistemática. Duazary. 2020 abril - junio; 17(2): 32 - 44. Doi: http://dx.doi.org/10.21676/2389783X.3231 \\ Recibido en julio 10 de 2019 \\ Aceptado en septiembre 04 de 2019 \\ Publicado en línea en diciembre 02 de 2019
}

\section{RESUMEN}

Palabras clave: teléfono

celular; personal de salud; contaminación; infección hospitalaria.
En el presente estudio se realizó una revisión sistemática para identificar microorganismos reportados como agentes contaminantes en teléfonos celulares de trabajadores de la salud que se desempeñan en ambientes clínicos. La investigación incluyó publicaciones entre 2008 y 2018 mediante búsqueda en seis bases de datos usando palabras clave como "nosocomial pathogen", "healthcare workers" y "cell pone", entre otras. Dos investigadores realizaron de forma independiente la búsqueda y revisión de artículos, cuya calidad se evaluó mediante lista de chequeo STROBE. De este modo, veintiún artículos fueron analizados. En todos estos se identificó contaminación en los celulares de los trabajadores de la salud, con una mediana de $84,3 \%$, resaltando la presencia de Staphylococcus aureus $(85,7 \%)$ y Escherichia coli $(61,9 \%)$. Se concluye que el uso de los teléfonos celulares en ambiente hospitalario por parte de los trabajadores de la salud constituye una fuente potencial de transmisión de microorganismos de interés clínico.

\section{ABSTRACT}

A systematic review was carried out to identify microorganisms reported as contaminating. Publications were included between 2008 and 2018, using keywords like "health workers" and "cell phone", among others. Research and review of articles. The papers quality is evaluated through the checklist STROBE. Twenty-one articles were analyzed. In all studies, the presence of contamination in the cell phones of health workers was identified, with a median of $84.3 \%$, highlighting the presence of Staphylococcus aureus (85.7\%) and Escherichia coli (61.9\%). It is concluded that the use of cell phones in the hospital environment by health workers is a potential source of transmission of microorganisms of clinical interest. 


\section{INTRODUCCIÓN}

El fenómeno de la globalización ha facilitado la incorporación de tecnologías de información y comunicación. Estas se han convertido en herramientas importantes en entornos clínicos que buscan mejorar la calidad en la atención en salud ${ }^{1}$. Sin embargo, la transición para adoptar estos cambios tecnológicos requiere de preparación para mitigar potenciales daños al paciente ${ }^{2}$.

Las características de los teléfonos inteligentes (ligereza, portabilidad y variedad de aplicaciones) los hacen los principales promotores de la incorporación de tecnología en la vida cotidiana ${ }^{3}$. El uso de estos dispositivos ha crecido de 3.200 millones a 5.400 millones en los últimos cinco años, lo que representa una tasa de crecimiento anual de $146 \% \%^{4,5}$.

Estudios realizados en trabajadores de la salud han documentado que cerca del $47 \%$ de estos usan el teléfono celular durante la jornada laboral en ambientes especiales como la unidad de cuidados intensivos $(\mathrm{UCl})$, y menos de la tercera parte lo descontaminan después de utilizarlo ${ }^{6,7}$. Como resultado, estos dispositivos no desinfectados tienen gran potencial de contaminación con diversos agentes patógenos. Se han reportado tasas de contaminación en teléfonos celulares de trabajadores de la salud que van desde el $47 \%$ hasta el $96 \%$, y entre el $9 \%$ y el $25 \%$ corresponden a microorganismos patógenos ${ }^{8}$. Esta conducta puede constituir un problema de salud pública considerando la posible contaminación cruzada mediante la transferencia de microorganismos pertenecientes a la flora normal de la piel, así como de potenciales agentes nosocomiales tales como bacterias, mohos y levaduras, entre otros ${ }^{9-11}$.

En ese orden de ideas, los teléfonos móviles se convierten en ambientes ideales para el crecimiento de los microorganismos, proporcionando la humedad y temperatura adecuada para su supervivencia y multiplicación. Para el año 2015, Ulger et al publicaron una revisión de la literatura que evaluó la potencial fuente de contaminación nosocomial a partir de los teléfonos móviles de trabajadores de la salud incluyendo artículos desde
2005 a $2013^{12}$. Sin embargo, se propone realizar una revisión sistemática ampliada a otras bases de datos que involucre nuevas publicaciones $y$ estudios latinoamericanos a fin de documentar recientes hallazgos bacteriológicos aislados a partir de teléfonos celulares de trabajadores de la salud. Para ello se propone la siguiente pregunta de investigación: ¿qué tipos de microorganismos bacterianos se pueden identificar como agentes contaminantes en teléfonos celulares de trabajadores de la salud que realizan actividades en ambientes clínicos?

\section{MATERIALES Y MÉTODOS}

\section{Tipo de estudio}

Se realizó una investigación secundaria tipo revisión sistemática $^{13,14}$.

\section{Criterios de elegibilidad de los estudios}

Como criterios de elegibilidad se consideraron investigaciones originales con diseño metodológico observacional o experimental, realizadas desde 2008 a 2018 en idiomas inglés, español y portugués. Fueron excluidos aquellos artículos cuya versión completa no estuviera disponible, así como resúmenes de eventos académicos y cartas al editor.

\section{Fuentes de información y estrategia de búsqueda}

La búsqueda de información se realizó utilizando términos MeSH y DeSC en seis bases de datos -PubMed, EBSCO, Web of Science, Embase, Scopus y LILACS - con las palabras "nosocomial pathogen", "fomites", "contamination", "health personnel", "healthcare workers", "cell pone", "mobile phone" y "mobile device". Mediante el uso de ecuaciones de búsqueda previamente definidas (Tabla 1), se recolectaron artículos aplicados con los filtros correspondientes a los criterios de elegibilidad (fecha e idioma). De forma preliminar, se revisaron títulos y resúmenes para descartar aquellos artículos que no fueran originales o que no correspondieran con la temática de la revisión. La búsqueda de información se llevó a cabo entre junio y septiembre de 2018. 
Tabla 1. Ecuaciones de búsqueda aplicadas en cada base de datos.

\begin{tabular}{|c|c|}
\hline Base de datos & Ecuación de búsqueda \\
\hline PubMed & $\begin{array}{c}\text { (((("nosocomial pathogen") OR fomites) OR contamination)) AND (("health personnel") OR } \\
\text { "healthcare workers")) AND }\end{array}$ \\
((("cell phone") OR "mobile phone") OR "mobile device")
\end{tabular}

\section{Selección de estudios}

De forma independiente, dos investigadoras recuperaron información en cada una de las bases de datos. En aquellos casos donde hubo discrepancia, una tercera investigadora revisó los artículos a fin de llegar a un consenso en la selección de literatura.

\section{Proceso de recopilación}

La información fue digitada en una base de datos en Excel y validada en el mismo programa para garantizar la consistencia por parte de las investigadoras. Posteriormente se extrajo y tabuló la información concerniente al cumplimiento de los objetivos, que finalmente fue analizada de forma cualitativa.

\section{Variables}

Dentro de las variables objeto de interés en la revisión sistemática se destacan los tipos de microorganismos aislados en teléfonos celulares, el área (intra u hospitalaria) donde se llevó a cabo el estudio, el tipo de trabajador de la salud, el tipo de estudio y el porcentaje de contaminación microbiana reportada, así como características de los artículos (país, año de publicación, número de autores), entre otras.

\section{Evaluación de riesgo de sesgos}

La calidad metodológica de los estudios recuperados se evaluó con las escala STROBE ${ }^{15}$, teniendo en cuenta que todos los artículos identificados correspondían a estudios de tipo observacional.

\section{Plan de análisis}

El análisis estadístico se realizó en el programa Microsoft $(C$ Excel 2018. Un análisis univariado permitió describir las características bibliométricas y las frecuencias de los microorganismos encontrados en los estudios. En este análisis se calcularon la frecuencia absoluta y el porcentaje para las variables cualitativas.

\section{Declaración sobre aspectos éticos}

Este estudio estuvo basado en la Declaración de Helsinki de 1975, conforme a la Ley 008430 de 1993 colombiana. Este trabajo consistió en una investigación sin riesgo debido a que se utilizaron técnicas de investigación de documentos retrospectivos, teniendo en cuenta que se trata de una revisión sistemática ${ }^{16}$. Adicionalmente, en consideración del tipo de investigación, se tuvieron en cuenta los derechos de autor de los artículos correspondientes, evitando el plagio de acuerdo con lo establecido en la Ley 1581 de 2012, el Decreto 
1377 de 2013, la Ley 23 de 1982, la Ley 44 de 1993 y el artículo 61 de la Constitución Política de $1991^{17-20}$.

\section{RESULTADOS}

Tras aplicar las ecuaciones de búsqueda, se identificó un total de 63 artículos. El proceso de filtración permitió excluir de las bases de datos de PubMed y Scopus artículos publicados en idiomas diferentes al inglés, el español y el portugués, así como los que no estaban disponibles en texto completo y que se habían publicado antes del año 2008 (segunda etapa de la búsqueda sistemática). De este modo quedaron 53 artículos que fueron revisados por título y resumen, con lo que se descartaron 14 (tercera etapa de la búsqueda). Así quedaron 39 artículos preseleccionados, los cuales se revisaron para identificar duplicados entre las diferentes bases de datos, y con ello se descartaron 11 artículos (cuarta etapa de la búsqueda sistemática). Finalmente, con la revisión de la versión completa de los artículos se descartaron 7 (cartas al editor $\mathrm{n}=2$, resúmenes en eventos científicos $n=2$, folletos informativos $n=3$ ), lo que dejó un total de 21 artículos para la revisión sistemática (Figura 1).
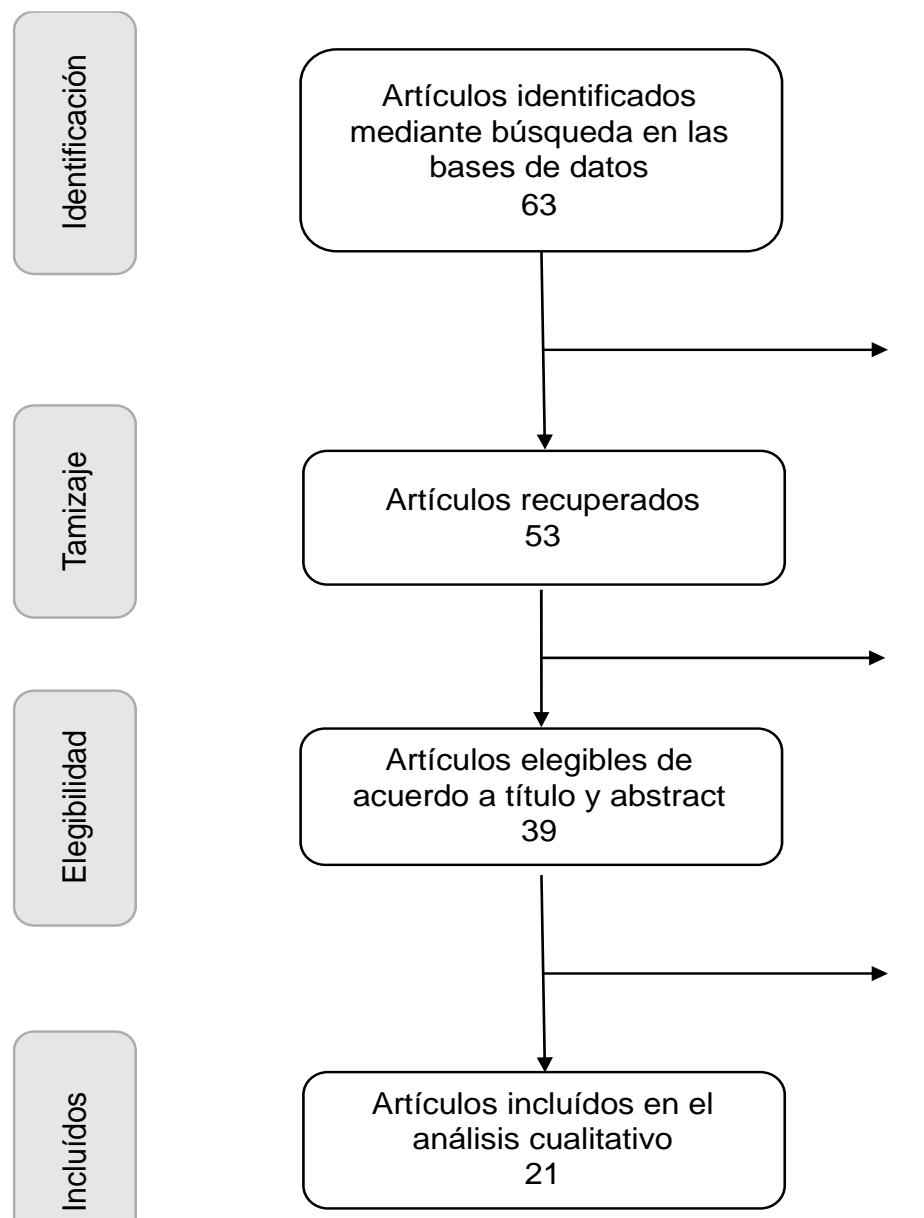

Artículos que no cumplían con criterios de selección (idioma-año de publicación)

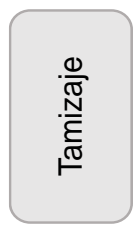
$\mathrm{n}=10$
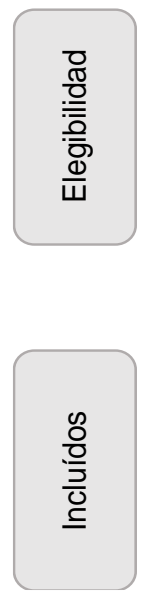

Artículos incluídos en el 21

Artículos excluídos por no corresponder a la temática del estudio $\mathrm{n}=14$

Cartas al editor $\mathrm{n}=2$

Resúmenes de Congresos $\mathrm{n}=2$

Folleto informativo $n=3$

Artículos duplicados $n=11$

Figura 1. Flujograma de estrategia de selección de artículos para la revisión sistemática.

Entre 2008 y 2018 se publicaron de 1 a 4 artículos por año, siendo el 2013 el pico máximo (4 publicaciones, 19\%). Así mismo, se evidenció que el país donde se realizaron publicaciones con mayor frecuencia fue India, con 4 (19\%). De acuerdo con los artículos de la presente revisión sistemática, se evaluaron en total 2.497 teléfonos celulares, en los cuales se identificó una frecuencia de contaminación que va desde $6,7 \%{ }^{21}$ hasta el $100 \%{ }^{22-}$ ${ }^{24}$. Esta presencia de contaminación microbiana en celulares tuvo una mediana de $84,3 \%$, con un rango intercuartílico de $71,8 \%$ a 94,5\% (Tabla 2). 
Tabla 2. Características de los artículos incluidos en la revisión sistemática.

\begin{tabular}{|c|c|c|c|c|}
\hline $\begin{array}{l}\text { Autores y año de } \\
\text { publicación }\end{array}$ & Revista & País & $\begin{array}{c}\text { Celulares } \\
\text { evaluados } \\
\text { (n) }\end{array}$ & $\begin{array}{c}\text { Grado de } \\
\text { contaminación } \\
\text { reportado (\%) }\end{array}$ \\
\hline Loyola et al $2018^{25}$ & Germs & Perú & 114 & 81,1 \\
\hline Karkee et al $2017^{26}$ & $\begin{array}{c}\text { Kathmandu University Medical } \\
\text { Journal }\end{array}$ & Nepal & 124 & 71,8 \\
\hline Chang et al $2017^{27}$ & PLoS One & China & 72 & 97,2 \\
\hline Kotris et al $2017^{9}$ & Med Glas (Zenica) & Croacia & 110 & 77,3 \\
\hline $\begin{array}{c}\text { Channabasappa et al } \\
2016^{28}\end{array}$ & J Evolution Med Dent Sci & India & 46 & 89 \\
\hline Loyola et al $2015^{6}$ & $\begin{array}{c}\text { American Journal of Infection } \\
\text { Control }\end{array}$ & Perú & 114 & 21 \\
\hline Heyba et al $2015^{29}$ & BMC Infectious Diseases & Kuwait & 213 & 73,3 \\
\hline Mark et al $2014^{30}$ & $\begin{array}{c}\text { International Journal of Clinical } \\
\text { Practice }\end{array}$ & $\begin{array}{l}\text { Irlanda del } \\
\text { Norte }\end{array}$ & 150 & 62 \\
\hline Nwankwo et al $2014^{31}$ & $\begin{array}{l}\text { Journal of Epidemiology and Global } \\
\text { Health }\end{array}$ & Nigeria & 112 & 94,6 \\
\hline Nirupa et al $2013^{22}$ & $\begin{array}{c}\text { International Journal of Pharma and } \\
\text { Bio Sciences Microbiology }\end{array}$ & India & 100 & 100 \\
\hline Lee et $a / 2013^{23}$ & J Hosp Med & Corea del sur & 203 & 100 \\
\hline $\begin{array}{c}\text { Garcia-Stuchi et al } \\
2013^{21}\end{array}$ & Cienc Cuid Saude & Brasil & 60 & 6,7 \\
\hline Reddy et al $2013^{32}$ & $\begin{array}{l}\text { Indian Journal of Public Health } \\
\text { Research \& Development }\end{array}$ & India & 82 & 82 \\
\hline Brady et al $2012^{33}$ & Telemed e-health & Escocia & 87 & 55 \\
\hline $\begin{array}{l}\text { Muñoz-Escobedo et al } \\
\qquad 2012^{24}\end{array}$ & $\begin{array}{l}\text { Archivos Venezolanos de } \\
\text { Farmacología y Terapéutica }\end{array}$ & México & 52 & 100 \\
\hline Mohammadi et al $2011^{34}$ & $\begin{array}{c}\text { African Journal of Microbiology } \\
\text { Research }\end{array}$ & Irán & 150 & 94 \\
\hline Brady et al $2011^{35}$ & Clin Microbiol Infect & Escocia & 102 & 84,3 \\
\hline Tekerekoglu et al $2011^{36}$ & $\begin{array}{c}\text { American Journal of Infection } \\
\text { Control } \\
\end{array}$ & Turquía & 67 & 86,5 \\
\hline Srikanth et al $2010^{37}$ & Journal of Infection Prevention & India & 51 & 94 \\
\hline Sadat-Ali et al $2010^{38}$ & $\begin{array}{c}\text { American Journal of Infection } \\
\text { Control }\end{array}$ & Arabia Saudí & 288 & 43,6 \\
\hline Ulger et al $2009^{39}$ & $\begin{array}{c}\text { Annals of Clinical Microbiology and } \\
\text { Antimicrobials }\end{array}$ & Turquía & 200 & 94,5 \\
\hline
\end{tabular}

Para evaluar el riesgo de sesgo de las publicaciones incluidas en la revisión sistemática, se aplicó la lista de chequeo STROBE. En la Tabla 3 se puede observar que los artículos cumplieron a cabalidad con estas características: resumen, antecedentes científicos, resultados, discusión de hallazgos claves. Por el contrario, tuvieron bajo cumplimiento apartados como: tipos de estudio (9,5\%), criterios de selección $(14,4 \%)$, variables de interés (19\%), fuentes de datos $(14,3 \%)$ y fuente de financiación de los estudios (19\%). 
Tabla 3. Evaluación de la calidad de los artículos de acuerdo con parámetros de la lista de chequeo STROBE.

\begin{tabular}{|c|c|c|}
\hline $\begin{array}{l}\text { Sección del } \\
\text { artículo }\end{array}$ & Característica revisada & $\begin{array}{l}\text { Nivel de cumplimiento } \\
(\%)\end{array}$ \\
\hline & (a) Se identifica el diseño del estudio en el título o el resumen & $9,5 \%$ \\
\hline $\begin{array}{l}\text { Título y } \\
\text { resumen }\end{array}$ & $\begin{array}{l}\text { (b) El resumen presenta una síntesis } \\
\text { estructurada y muy informativa del artículo, } \\
\text { teniendo en cuenta los puntos desarrollados en } \\
\text { la lista de comprobación que sigue }\end{array}$ & $100 \%$ \\
\hline $\begin{array}{l}\text { Antecedentes/ } \\
\text { fundamentos }\end{array}$ & $\begin{array}{l}\text { Explica los antecedentes científicos y los fundamentos del } \\
\text { estudio }\end{array}$ & $100 \%$ \\
\hline Objetivos & $\begin{array}{l}\text { Consigna los objetivos específicos y cualquier hipótesis } \\
\text { preespecificada }\end{array}$ & $95,2 \%$ \\
\hline \multirow[t]{2}{*}{$\begin{array}{c}\text { Diseño del } \\
\text { estudio }\end{array}$} & Presenta los elementos clave del diseño del estudio & $47,6 \%$ \\
\hline & $\begin{array}{l}\text { Describe lugares y fechas, definiendo los periodos de recogida } \\
\text { de datos }\end{array}$ & $52,4 \%$ \\
\hline Participantes & $\begin{array}{c}\text { Explica los criterios de inclusión y exclusión, la fuente y los } \\
\text { métodos de selección de los participantes }\end{array}$ & $14,3 \%$ \\
\hline Variables & $\begin{array}{c}\text { Proporciona una lista y define claramente todas las variables } \\
\text { de interés (categorizaciones o agrupaciones) }\end{array}$ & $19 \%$ \\
\hline Medidas & Proporciona las fuentes de datos & $14,3 \%$ \\
\hline $\begin{array}{l}\text { Tamaño de la } \\
\text { muestra }\end{array}$ & Explica cómo se llegó al tamaño de la muestra & $9,5 \%$ \\
\hline $\begin{array}{l}\text { Métodos } \\
\text { estadísticos }\end{array}$ & Describe los análisis estadísticos aplicados al conjunto de datos & $61,9 \%$ \\
\hline \multirow[t]{2}{*}{$\begin{array}{c}\text { Datos } \\
\text { descriptivos }\end{array}$} & $\begin{array}{l}\text { Describe las características de los participantes en el estudio } \\
\text { (datos demográficos, clínicos o sociales) }\end{array}$ & $90,7 \%$ \\
\hline & $\begin{array}{c}\text { Informa el número de sucesos del evento o de medidas } \\
\text { resumen (frecuencia del evento) }\end{array}$ & $100 \%$ \\
\hline Hallazgos clave & $\begin{array}{c}\text { Resume los hallazgos más importantes en relación con las } \\
\text { hipótesis del estudio }\end{array}$ & $100 \%$ \\
\hline Limitaciones & $\begin{array}{l}\text { Discute las limitaciones de estudio, teniendo en cuenta las } \\
\text { fuentes potenciales de sesgo o de falta de precisión y los } \\
\text { problemas que pudieran derivarse por la multiplicidad de } \\
\text { análisis, exposiciones y resultados estudiados. Discute tanto a } \\
\text { la dirección como la magnitud de los potenciales sesgos }\end{array}$ & $28,5 \%$ \\
\hline Interpretación & $\begin{array}{c}\text { Da una interpretación cautelosa general de los resultados } \\
\text { teniendo en cuenta los objetivos, las limitaciones, la } \\
\text { multiplicidad de análisis, los resultados de estudios similares y } \\
\text { otras pruebas pertinentes }\end{array}$ & $90,5 \%$ \\
\hline Generalización & $\begin{array}{l}\text { Discute la generalización (validez externa) de los resultados del } \\
\text { estudio }\end{array}$ & $9,5 \%$ \\
\hline Financiamiento & $\begin{array}{c}\text { Da la fuente de financiación y el papel de los patrocinadores } \\
\text { para el presente estudio y, en su caso, para el estudio original } \\
\text { en el que se basa el presente artículo }\end{array}$ & $19,0 \%$ \\
\hline
\end{tabular}


Una amplia gama de bacterias fueron aisladas a partir de los teléfonos celulares. Por su compromiso con enfermedades nosocomiales, dentro del grupo de las Gram positivas se destacan Staphylococcus aureus (85,7\%) Enterococcus faecalis (9,5\%) y, en el grupo de las Gram negativas, Escherichia coli (61,9\%), Klebsiella pneumoniae (23,8\%) y Pseudomonas aeruginosa (38,1\%) (Tabla 4). En cuanto al área asistencial donde más se han adelantado investigaciones, la UCl predominó $(38,1 \%)$, seguida de cirugía general $(33,1 \%)$. Finalmente, el personal de salud al que más se le ha realizado aislamientos bacteriológicos en sus teléfonos celulares han sido médicos $(57,1 \%)$ y enfermeras (42,9\%) (Tabla 5).

Tabla 4. Frecuencia de aislamiento de bacterias a partir de teléfonos celulares de trabajadores de la salud.

\begin{tabular}{|c|c|c|c|}
\hline Gram positivas* & n (\%) & Gram negativas* & $n(\%)$ \\
\hline Staphylococcus aureus & $18(85,7)$ & Escherichia coli & $13(61,9)$ \\
\hline Staphylococcus coagulasa (-) & $7(33,3)$ & Klebsiella pneumoniae & $5(23,8)$ \\
\hline Staphylococcus epidermidis & $2(9,5)$ & Klebsiella oxytoca & $1(4,8)$ \\
\hline Staphylococcus MSSA & $1(4,8)$ & Klebsiella sp. & $5(23,8)$ \\
\hline Staphylococcus MRSA & $1(4,8)$ & Pseudomonas aeruginosa & $8(38,1)$ \\
\hline Staphylococcus sp. & $1(4,8)$ & Pseudomonas putida & $1(4,8)$ \\
\hline Streptococcus agalactiae & $1(4,8)$ & Pseudomonas stutzeri & $1(4,8)$ \\
\hline Streptococcus viridans & $2(9,5)$ & Pseudomonas sp. & $3(14,3)$ \\
\hline Streptococcus faecium & $1(4,8)$ & Acinetobacter baumannii & $1(4,8)$ \\
\hline Streptococcus mitis & $1(4,8)$ & Acinetobacter ursingii & $1(4,8)$ \\
\hline Streptococcus salivarius & $1(4,8)$ & Acinetobacter sp. & $7(33,3)$ \\
\hline Streptococcus sp. & $6(28,6)$ & Enterobacter cloacae & $2(9,5)$ \\
\hline Enterococcus faecalis & $2(9,5)$ & Enterobacter sp. & $3(14,3)$ \\
\hline Enterococcus faecium & $1(4,8)$ & Proteus sp. & $3(14,3)$ \\
\hline Enterococcus sp. & $7(33,3)$ & Salmonella sp. & $1(4,8)$ \\
\hline Micrococcus sp. & $6(28,6)$ & Shigella sp. & $1(4,8)$ \\
\hline Bacillus sp. & $6(28,6)$ & Moraxella sp. & $1(4,8)$ \\
\hline Corynebacterium sp. & $3(14,3)$ & Citrobacter koseri & $1(4,8)$ \\
\hline Difteroides & $1(4,8)$ & Bacteroides vulgaris & $1(4,8)$ \\
\hline Sarcina & $1(4,8)$ & Burkholderia cepacia & $1(4,8)$ \\
\hline \multirow{3}{*}{\multicolumn{2}{|c|}{$\begin{array}{l}\text { MSSA: meticilinosensible } \\
\text { MSRA: meticilinorresistente }\end{array}$}} & Rhizobium radiobacter & $1(4,8)$ \\
\hline & & Sphingomonas paucimobilis & $1(4,8)$ \\
\hline & & Enterobacterias en general & $4(19,0)$ \\
\hline
\end{tabular}

*Porcentajes calculados considerando un total de 21 artículos incluidos en la revisión sistemática. 
Tabla 5. Área intrahospitalaria y tipo de trabajador de la salud donde se evaluó presencia de bacterias.

\begin{tabular}{|c|c|}
\hline Área intrahospitalaria & $\mathrm{n}(\%)$ \\
\hline Unidad de cuidados intensivos & $8(38,1)$ \\
\hline Unidad de cuidados intensivos pediátrica-neonatal & $2(9,5)$ \\
\hline Cirugía general & $7(33,3)$ \\
\hline Cirugía ortopédica & $1(4,8)$ \\
\hline Unidad de hemodiálisis & $1(4,8)$ \\
\hline Neurología & $1(4,8)$ \\
\hline Dermatología & $1(4,8)$ \\
\hline Ginecología & $1(4,8)$ \\
\hline Ortopedia & $1(4,8)$ \\
\hline Medicina general & $1(4,8)$ \\
\hline Sin especificar & $1(4,8)$ \\
\hline \multicolumn{2}{|l|}{ Personal de salud } \\
\hline Médicos & $12(57,1)$ \\
\hline Enfermeras & $9(42,9)$ \\
\hline Cirujanos & $3(14,3)$ \\
\hline Técnicos-auxiliares de enfermería & $2(9,5)$ \\
\hline Pediatras & $2(9,5)$ \\
\hline Nutricionistas & $1(4,8)$ \\
\hline Fisioterapeutas & $1(4,8)$ \\
\hline Odontólogos & $1(4,8)$ \\
\hline Radiólogos & $1(4,8)$ \\
\hline Farmaceutas & $1(4,8)$ \\
\hline Personal UCI & $1(4,8)$ \\
\hline Docentes área de la salud & $3(14,3)$ \\
\hline Estudiantes área de la salud & $2(9,5)$ \\
\hline Personal de salud en general & $8(38,1)$ \\
\hline
\end{tabular}

\section{DISCUSIÓN}

Desde el momento de la incorporación del teléfono para uso en ambientes clínicos, investigadores han considerado este medio de comunicación como potencial fuente de contaminación microbiológica entre pacientes y personal de salud, así como entre pacientes y sus visitantes ${ }^{40}$. Esta investigación tuvo como propósito identificar en los artículos científicos publicados entre 2008 y 2018 las bacterias reportadas como agentes contaminantes en teléfonos celulares de trabajadores de la salud que realizan actividades en ambientes clínicos. Mediante la presente revisión sistemática se obtuvieron 21 artículos en los cuales se encontró evidencia de contaminación microbiana que osciló entre $6,7 \%$ y $100 \%$, con una mediana de $84,3 \%$. Estos resultados fueron consistentes con los reportados por Ulger et al, quienes en 2015 llevaron a cabo una revisión de la literatura en la cual evaluaron a los trabajadores de salud como potenciales fuentes de contaminación en ambiente hospitalario y encontraron que la prevalencia de agentes nosocomiales aislados de los teléfonos celulares osciló entre $10 \%$ y $100 \%{ }^{12}$.

La mediana de contaminación bacteriana reportada en el presente trabajo es similar a la encontrada por la mayoría de los estudios revisados ${ }^{9,25,37,39,26-}$ 29,31,32,35,36. No obstante, el nivel de contaminación reportado fue distinto al identificado por una minoría de autores ${ }^{6,21}$. La discrepancia con Loyola et $a l^{6}$ radica en que su trabajo se centró en la identificación de enterobacterias, quedando por fuera un porcentaje importante de microorganismos de interés clínico. En el caso del estudio de García-Stuchi et al ${ }^{21}$, quienes reportaron 
una frecuencia de contaminación del 6,7\%, la más baja identificada, es posible que esto se debiera a la utilización de medios selectivos para el aislamiento primario de microorganismos.

El microorganismo Gram positivo identificado con mayor frecuencia en los 21 estudios incluidos fue el S. aureus $(85,7 \%)$. Este dato es consistente con lo reportado por Ulger et al en la revisión de la literatura de 2015, quienes encontraron este agente etiológico en 26 de 39 estudios revisados $(66,7 \%)$, los cuales habían sido publicados entre 2005 y $2013^{12}$. En el mundo se estima que cerca del $30 \%$ de la población es portadora asintomática de esta bacteria, la cual hace parte de la microbiota nasal, de donde fácilmente se puede transmitir a las manos y a los dispositivos móviles del personal de la salud y causar infecciones nosocomiales ${ }^{41}$. Otra situación preocupante frente a este agente es la resistencia generada a la meticilina, pues un metaanálisis de reciente publicación evidenció que la prevalencia media de portadores de $S$. aureus meticilinorresistente es de 3,5\% de los 4,5 billones individuos de la región de Asia-Pacífico, lo que constituye un problema de salud pública emergente ${ }^{42}$.

Para el caso de los microorganismos Gram negativos, la $E$. coli predominó con un $61,9 \%$. Esta bacteria de la familia de las enterobacterias, aunque es un comensal que usualmente se utiliza como un indicador de contaminación fecal, tiene dentro de sus variedades cepas patógenas de interés clínico tales como: productora de toxina Shiga, enteropatogénica, enterotoxigénica, enteroagregativa y enterohemorrágica. Estas se han asociado a una amplia variedad de enfermedades que en algunos casos son letales, ocasionando hasta dos millones de muertes al año. Debido a que la $E$. coli hace parte de la microbiota del intestino de los humanos, es razonable que esté en contacto frecuente con antibióticos y con ello exhiba diferentes niveles de resistencia a estos ${ }^{43}$.

El área intrahospitalaria en la cual se realizó el estudio con mayor frecuencia fue en las zonas con asepsia como UCl y salas de cirugía general. Llama la atención que en estos espacios, si bien se debe mantener total asepsia y esterilidad, y a pesar de tener normas de bioseguridad, no se cuente con una prohibición explícita a la utilización de teléfonos celulares ${ }^{44}$. Autores que han identificado esta problemática proponen estrategias tales como restricción del teléfono móvil en unidades hospitalarias de alto riesgo, limpieza periódica de estos dispositivos, técnicas de higiene de manos por parte de los trabajadores de la salud y los pacientes, uso de audífonos bluetooth y cubiertas antibacterianas para los dispositivos móviles, entre otras $^{12}$.

Dentro del personal de salud en el que se han centrado las investigaciones sobre contaminación bacteriana a partir de teléfonos celulares están médicos y enfermeras $\quad(57,1 \%$ y $42,9 \%$ respectivamente). Esto se explica porque son los profesionales con predominio en las instituciones de salud y, para el caso de enfermería, porque permanecen junto al paciente las 24 horas $y$ desarrollan una relación estrecha con él ${ }^{45}$. Ahora bien, llama la atención que los estudios se han interesado por documentar además esta situación en el personal que realiza actividad docenciaasistencial $(14,3 \%)$ y en estudiantes del área de la salud $(9,5 \%)$. Vale la pena destacar esto último considerando que los dispositivos móviles se han ido incorporando en el proceso de aprendizaje de la educación superior ${ }^{46}$. Estudios que han evaluado la contaminación microbiana comparando teléfonos celulares de estudiantes y docentes del área de la salud (odontología) versus ingeniería, si bien no han encontrado diferencias en las prácticas de desinfección en los dispositivos, sí reportan mayores tasas de contaminación bacteriana en los estudiantes de odontología $(p<0,01)^{5}$.

Mediante la aplicación de la herramienta STROBE se evaluó la calidad de los artículos de la presente revisión sistemática ${ }^{14}$, y se encontró que menos del $10 \%$ de las publicaciones reportaron el tipo de estudio que había sido aplicado. Así mismo, detalles metodológicos claves tales como características de participantes, variables de interés en el estudio y la estimación del tamaño de muestra fueron pobremente reportadas por los autores. Esto constituye una debilidad de la calidad metodológica de los estudios analizados ${ }^{15}$.

Dentro de las limitaciones del presente trabajo se destaca que todos los estudios analizados fueron de tipo observacional. Si bien estos son una herramienta importante para conocer las 
características generales de una población o grupo de interés, su diseño epidemiológico es susceptible a presencia de sesgos de información y de selección. Por lo tanto, la calidad de la evidencia puede verse comprometida y restringe la inferencia de dichos resultados ${ }^{47}$. Así mismo, se ha identificado que el interés de los investigadores se ha orientado más recientemente a documentar la resistencia bacteriana a los fármacos de uso clínico. No obstante, esta variable no ha sido documentada en todos los estudios revisados, por lo que se propone para futuras revisiones abordar como eje central la multidrogorresitencia de las bacterias aisladas a partir de teléfonos celulares ${ }^{48,49}$. De igual forma, un elemento que vale la pena explorar en futuros estudios es la presencia de otros agentes tales como virus y hongos, entre otros, que pueden comportarse como patógenos nosocomiales de interés clínico presentes en los teléfonos celulares $^{10,50}$.

Por otro lado, se plantea como fortaleza que se realizó una búsqueda en seis bases de datos del área de la salud en las cuales se aplicaron diferentes ecuaciones, con lo que se amplió el espectro de identificación de artículos. De igual manera, se consideraron varios idiomas para incluir a todos aquellos pertinentes para la pregunta de investigación del estudio (inglés, español, portugués). Esta revisión sistemática constituye así la primera que a la fecha se ha adelantado para establecer el grado de contaminación bacteriológica que se puede presentar en el área asistencial a partir del contacto con dispositivos móviles de uso personal.

En conclusión, el uso de los teléfonos celulares en ambiente hospitalario por parte del personal que presta servicios de salud constituye una fuente potencial de transmisión de microorganismos de interés clínico. Teniendo en cuenta la alta frecuencia de hallazgo de estos agentes bacteriológicos en los teléfonos móviles del personal de salud, se hace necesario aumentar la rigurosidad y vigilancia en cuanto al cumplimiento de protocolos para el uso de estos dispositivos en áreas clínicas.

\section{DECLARACIÓN DE CONFLICTOS DE INTERESES}

Los autores manifiestan que durante la ejecución del trabajo y la redacción del manuscrito no han incidido intereses o valores distintos a los que usualmente tiene la investigación.

\section{CONTRIBUCIÓN DE LOS AUTORES}

Primer autor: trabajo de campo, diseño metodológico y redacción.

Segundo autor: trabajo de campo y construcción base de datos.

Tercer autor: trabajo de campo y construcción base de datos.

Cuarto autor: redacción.

\section{REFERENCIAS BIBLIOGRÁFICAS}

1. Alotaibi YK FF. The impact of health information technology on patient safety. Rev Artic. 2017; 38(12): $\quad 1173-80 . \quad$ Doi: https://doi.org/10.15537/smj.2017.12.20631.

2. Coiera E, Aarts J, Kulikowski C. The dangerous decade. J Am Med Inf Assoc. 2012; 19: 2-5. Doi: https://doi.org/10.1136/amiajnl-2011-000674.

3. Cedeño-Luna R, Alcívar-Vaca K, Ponce-Vásquez D. Observations about mobile devices. Dom Cien [revista en la internet]. 2017; 3(4): 89-103. Disponible en: http://dominiodelasciencias.com/ojs/index.php /es/index.

4. Chopra RC. The Association between Cellular Phone Usage and Brain Neoplasms [internet]. 2016; 103-15. Disponible en: http://www.scirp.org/journal/odem.

5. Hosseini R, Hosseini R, Moradi M. Evaluation of the cell phone microbial contamination in dental and engineering schools: Effect of antibacterial spray. J Epidemiol Glob Health [revista en la internet]. 2017; 1-6. Doi: https://doi.org/10.1016/j.jegh.2017.10.004.

6. Loyola S, Gutiérrez L, Horna G, Petersen K, Agapito J, Osada J. Extended-spectrum $\beta$ lactamase-producing Enterobacteriaceae in cell phones of health care workers from Peruvian pediatric and neonatal intensive care units. Am J Infect Control [revista en la internet]. 2016; 44(8): 1-7. Doi: http://dx.doi.org/10.1016/j.ajic.2016.02.020.

7. Zakai S, Mashat A, Abumohssin A, Samarkandi A, 
Almaghrabi $B$, Barradah $\mathrm{H}$, et al Bacterial contamination of cell phones of medical students at King Abdulaziz University, Jeddah, Saudi Arabia. J Microsc Ultrastruct [revista en la internet]. 2016; 4(3): 143-6. Disponible en: http://www.jmau.org/text.asp?2016/4/3/143/ 224860.

8. Brady RRW, Verran J, Damani NN, Gibb AP. Review of mobile communication devices as potential reservoirs of nosocomial pathogens. J Hosp Infect [revista en la internet]. 2009; 71(4): 295-300.

Doi: http://dx.doi.org/10.1016/j.jhin.2008.12.009.

9. Kotris I, Drenjančević D, Talapko J, Bukovski S. Identification of microorganisms on mobile phones of intensive care unit health care workers and medical students in the tertiary hospital. Med Glas. 2017; 14(1): 85-90. Doi: https://doi.org/10.17392/878-16.

10. Pillet S, Berthelot P, Mory O, Gay C, Viallon A, Lucht $F$, et al Contamination of healthcare workers' mobile phones by epidemic viruses. Clin Microbiol Infect [revista em la internet]. 2016; 22(5): 456.e1-456.e6. Doi: http://dx.doi.org/10.1016/j.cmi.2015.12.008.

11. Kordecka A, Cecylia $Ł$, Ku W. Isolation frequency of Candida present on the surfaces of mobile phones and handsx. BMC Infect Dis. 2016; 16(238): $\quad 1-7 . \quad$ Doi: https://doi.org/10.1186/s12879-016-1577-0.

12. Ulger F, Dilek A, Esen S, Sunbul M, Leblebicioglu $\mathrm{H}$. Are healthcare workers' mobile phones a potential source of nosocomial infections? Review of the literature. J Infect Dev Ctries. 2015; 9(10): 1046-53. Doi: https://doi.org/10.3855/jidc.6104.

13. Oscar BG. Revisiones sistemáticas de la literatura. Rev Colomb Gastroenterol [revista en la internet]. 2005; 20 (1): 60-9. Disponible en: http://www.scielo.org.co/pdf/rcg/v20n1/v20n1 a09.pdf.

14. Donis J. Tipos de diseños de los estudios clínicos y epidemiológicos. Av en Biomed [revista en la internet]. 2013; 2(2): 76-99. Disponible en: http://www.redalyc.org/pdf/3313/3313279890 05.pdf.
15. Vandenbroucke JP, Elm E Von, Altman DG, Gøtzsche PC, Mulrow CD, Pocock SJ, et al Strengthening the Reporting of Observational Studies in Epidemiology (STROBE): Explanation and elaboration. Int J Surg [revista en la internet]. 2014; 12(12): 1500-24. Doi: http://dx.doi.org/10.1016/j.ijsu.2014.07.014.

16. Colombia. Ministerio de Salud. Resolución 8430 de 1993 por la cual se establecen las normas científicas, técnicas y administrativas para la investigación en salud [internet]. Bogotá: EI Ministerio; 1993. Disponible en: https://www.minsalud.gov.co/sites/rid/Lists/Bi bliotecaDigital/RIDE/DE/DIJ/RESOLUCION8430-DE-1993.PDF.

17. Colombia. Congreso de la República. Ley Estatutaria 1581 de 2012 por la cual se dictan disposiciones generales para la protección de datos personales. Diario Oficial, 48587 (Oct. 18 2012). Disponible en: http://www.secretariasenado.gov.co/senado/b asedoc/ley_1581_2012.html.

18. Colombia. Ministerio de Comercio, Industria y Turismo. Decreto 1377 de 2013 por el cual se reglamenta parcialmente la Ley 1581 de 2012. Diario Oficial, 48834 (Jun. 27 2013). Disponible en:

http://wsp.presidencia.gov.co/Normativa/Decr etos/2013/Documents/JUNIO/27/DECRETO\%2 01377\%20DEL\%2027\%20DE\%20JUNIO\%20DE\% 202013.pdf.

19. Colombia. Congreso de la República. Ley 23 de 1982 sobre derechos de autor. Bogotá: EI Congreso; $1982 . \quad$ Disponible en: http://derechodeautor.gov.co/documents/101 81/182597/23.pdf/a97b8750-8451-4529-ab87bb82160dd226.

20. Colombia. Congreso de la República. Ley 44 de 1993 por la cual se modifica y adiciona la Ley 23 de 1982 y se modifica la Ley 29 de 1994. Diario oficial, 40740 (Feb. 5 1993). 1993. Disponible en: http://derechodeautor.gov.co/documents/101 81/182597/44.pdf/7875d74e-b3ef-4a8a-8661$704823 b 871 b 5$.

21. García-Stuchi R, De-Almeida-Silva C, Soares B, Arreguy-Sena C. Bacterial and fungal contamination of mobile phones belonging to 
the health team of a hospital in Minas Gerais state. Cienc Cuid Saude. 2013; 12(4): 760-7.

22. Nirupa S, Bhabu V, Jeya M. Can mobile phones act as vehicles transmitting nosocomial infections? Int J Pharma Bio Sci Microbiol [revista en la internet]. 2013; 4(1): 859-64. Disponible en: https://ijpbs.net/abstract.php?article=MTk4Nw

23. Lee YJ, Yoo C, Lee $C$, Chung HS. Contamination Rates Between Smart Cell Phones and NonSmart Cell Phones of Healthcare Workers. J Hosp Med. 2013; 8(3): 2011-4. Doi: https://doi.org/10.1002/jhm.2011.

24. Muñoz-Escobedo J, Varela-Castillo L, ChávezRomero P, Becerra-Sánchez A, Moreno-García $M$. Bacterias patógenas aisladas de teléfonos celulares del personal y alumnos de la Clínica Multidisciplinaria (CLIMUZAC) de la Unidad Académica de Odontología de la UAZ. Arch Venez Farmacol y Ter [revista en la internet]. 2012; 31(2): 23-31. Disponible en: http://www.redalyc.org/articulo.oa?id=559249 50005.

25. Loyola S, Gutiérrez L, Severino N, Tamariz J. Multidrug-resistant bacteria isolated from cell phones in five intensive care units : Exploratory dispersion analysis. Germs. 2018; 8(2): 85-91. Doi:

https://doi.org/10.18683/germs.2018.1135.

26. Karkee $P$, Sk M, Humagain P, Thaku N, Timilsina B. Mobile Phone : A Possible Vector of Bacterial Transmission in Hospital Setting. Kathmandu Uni Med [revista en la internet]. 2017; 59(3): 217-21. Disponible en: https://www.ncbi.nlm.nih.gov/pubmed/30353 896.

27. Chang C, Chen S, Lu J, Chang C. Nasal colonization and bacterial contamination of mobile phones carried by medical staff in the operating room. PLoS One. 2017; 12(5): e0175811.

Doi: https://doi.org/10.1371/journal.pone.0175811.

28. Channabasappa S, Venkatarao G, Dharmappa S. A study of microbiological contamination of mobile phones in operating room personnel and efficacy of decontamination with $2 \%$ isopropyl alcohol. J Evol Med Dent Sci. 2016; 5(10): 43841. https://doi.org/10.14260/jemds/2016/100.

29. Heyba M, Ismaiel $M$, Alotaibi A, Mahmoud $M$, Baqer $\mathrm{H}$, Safar $\mathrm{A}$, et al Microbiological contamination of mobile phones of clinicians in intensive care units and neonatal care units in public hospitals in Kuwait. BMC Infect Dis. 2015; 15(434): 1-9. Doi: http://dx.doi.org/10.1186/s12879-015-1172-9.

30. Mark D, Leonard C, Breen H, Graydon R, Gorman $\mathrm{CO}$, Kirk S. Mobile phones in clinical practice: reducing the risk of bacterial contamination. Int J Clin Pr. 2014; 68(9): 1060-4. Doi: https://doi.org/10.1111/ijcp.12448.

31. Nwankwo EO, Ekwunife N, Mofolorunsho KC. Nosocomial pathogens associated with the mobile phones of healthcare workers in a hospital in Anyigba, Kogi state, Nigeria. J Epidemiol Glob Health. 2014; 4: 135-40. Doi: https://doi.org/10.1016/j.jegh.2013.11.002.

32. Reddy S, Tapashetti S, Prasad B, Kumar V, Kallapanavar N. Microbiological Profile of Organisms Cultured from Medical Staff Mobile Phones in ICU Units at a Tertiary Care Hospital K. Indian J Public Heal Res Dev. 2013; 4(4): 34-7. Doi: https://doi.org/10.5958/j.09765506.4.4.137.

33. Brady R, Chitnis S, Stewart R, Graham C, Yalamarthi S, Morris K. NHS Connecting for Health: Healthcare Professionals, Mobile Technology, and Infection Control. Telemed ehealth. 2012; 18(4): 289-92. Doi: https://doi.org/10.1089/tmj.2011.0147.

34. Mohammadi-Sichani $M$, Karbasizadeh $V$. Bacterial contamination of healthcare workers mobile phones and efficacy of surface decolonization techniques. African J Microbiol Res. 2011; 5(30): 5415-8. Doi: https://doi.org/10.5897/AJMR11.1062.

35. Brady R, Hunt A, Visvanathan A, Rodrigues M, Graham C, Rae C, et al Mobile phone technology and hospitalized patients: a cross-sectional surveillance study of bacterial colonization, and patient opinions and behaviours. Clin Microbiol 
Infect. 2011; 17(6): 830-5. Doi: https://doi.org/10.1111/j.1469-

0691.2011.03493.x.

36. Tekerekoglu M, Duman Y, Serindag A, Cuglan S, Kaysadu $H$, Tunc $E$, et al Do mobile phones of patients, companions and visitors carry multidrug-resistant hospital pathogens ? Am J Infect Control. 2011; 39(5): 379-81. Doi: https://doi.org/10.1016/j.ajic.2010.10.026.

37. Srikanth P, Rajaram E, Sudharsanam $S$, Lakshmanan A, Sivamurugan U, Mariappan S, et al Mobile phones: emerging threat for infection control. J Infect Prev. 2010; 11(3): 87-90. Doi: https://doi.org/10.1177/1757177410364866.

38. Sadat-Ali M, Al-Omran AK, Azam Q, Bukari H. Bacterial flora on cell phones of health care providers in a teaching institution. Am J Infect Control Epidemiol. 2010; 38(5): 404-5. Doi: http://dx.doi.org/10.1016/j.ajic.2009.08.007.

39. Ulger F, Esen S, Dilek A, Yanik K, Gunaydin M, Leblebicioglu $\mathrm{H}$. Are we aware how contaminated our mobile phones with nosocomial pathogens? Ann Clin Microbiol Antimicrob. 2009; 8(7): 1-4. Doi: https://doi.org/10.1186/1476-0711-8-7.

40. Aronson S. The Lancet on the telephone 18761975. Med Hist. 1977; 21: 69-87. Doi: https://www.ncbi.nlm.nih.gov/pmc/articles/P MC1081896/.

41. Sakr A, Brégeon F, Mège J, Rolain J, Gobert AP. Staphylococcus aureus nasal colonization: an update on mechanisms, epidemiology, risk factors, and subsequent infections. Front Microbiol. 2018; 9: 1-15. Doi: https://doi.org/10.3389/fmicb.2018.02419.

42. Wong JWH, Tang A, Wi V, Wong SYS, Read JM, Kwok KO. Prevalence and risk factors of community- associated methicillin-resistant Staphylococcus aureus carriage in Asia-Pacific region from 2000 to 2016 : a systematic review and meta-analysis. 2018; 1489-501. Doi: https://doi.org/10.2147/CLEP.S160595.

43. Jang J, Hur H, Sadowsky MJ, Byappanahalli MN, Yan T, Ishii S. Environmental Escherichia coli: ecology and public health implications - a review. J Appl Microbiol. 2017; 123: 570-81. Doi: https://doi.org/10.1111/jam.13468.

44. Manning M, David J, Sparnon E, Ballard R. iPads, droids, and bugs: infection prevention for mobile handheld devices at the point of care. Am J Infect Control. 2013; 41: 1073-6. Doi: https://doi.org/10.1016/j.ajic.2013.03.304.

45. Triana M. La empatía en la relación enfermerapaciente. Av Enferm. 2017; 35(2): 121-2. Doi: http://dx.doi.org/10.15446/av.enferm.v35n2.6 6941.

46. Ahmed S, Parsons D. Computers \& Education Abductive science inquiry using mobile devices in the classroom. Comput Educ. 2013; 63: 62-72. Doi:

http://dx.doi.org/10.1016/j.compedu.2012.11. 017.

47. Hernández M, Garrido F, Martínez E. Sesgos en estudios epidemiológicos. Salud Pública Mex [revista en la internet]. 2000; 42(5): 438-46. Disponible en: https://www.scielosp.org/article/ssm/content/ raw/?resource_ssm_path=/media/assets/spm/ v42n5/3995.pdf.

48. Banawas $S$, Abdel-hadi A, Alaidarous $M$, Alshehri B, Aziz A, Dukhyil B, et al Multidrug-Resistant Bacteria Associated with Cell Phones of Healthcare Professionals in Selected Hospitals in Saudi Arabia. Can J Infect Dis Med Microbiol. 2018; 2018: 1-7. Doi: https://doi.org/10.1155/2018/6598918.

49. Khashei R, Ebrahim-Saraie HS, Hadadi M, Ghayem M SH. The occurrence of nosocomial pathogens on cell phones of healthcare workers in an Iranian tertiary care hospital. Infect Disord Drug Targets. 2018.2 Doi: https://doi.org/10.2174/187152651866618083 0165732.

50. Cavari Y, Kaplan O, Zander A, Hazan G, ShemerAvni $Y$, et al Healthcare workers mobile phone usage: A potential risk for viral contamination. Surveillance pilot study. Infect Dis. 2016; 48(6): 432-5.

Doi: https://doi.org/10.3109/23744235.2015.11339 26. 